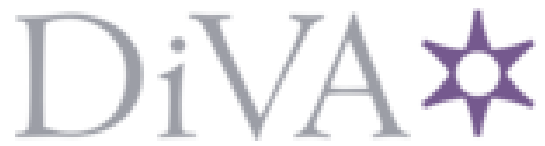

http://www.diva-portal.org

Preprint

This is the submitted version of a paper published in IEEE pervasive computing.

Citation for the original published paper (version of record):

Hazas, M. (2021)

Roombas and Landroids: Do Domestic Service Robots Save Energy?

IEEE pervasive computing, 20(2): 54-57

https://doi.org/10.1109/MPRV.2021.3067375

Access to the published version may require subscription.

N.B. When citing this work, cite the original published paper.

Permanent link to this version:

http://urn.kb.se/resolve?urn=urn:nbn:se:uu:diva-44678o 
Department: Smart Home

Author:

Mike Hazas

Uppsala University

Editors:

Jeannie Albrecht (jeannie@cs.williams.edu)

A.J. Brush (ajbrush@microsoft.com)

Mike Hazas (mike.hazas@it.uu.se)

\section{Roombas and Landroids: Do domestic service robots save energy?}

The past two decades have a seen a rise of autonomous robots in households. In particular, robotic vacuum cleaners and lawn mowers have become much more accessible and mainstream, and are the two most popular domestic service robots. (Roomba and Landroid are trade names for iRobot's vacuum and WORX's mower, respectively.) As with many smart home technologies, an often-touted benefit is that they can save energy - and many informal analyses are posted online. But whether and how they save energy, depends on how they are meant to be used, and how they are actually used.

"There is clearly a risk that the energy-savings promise of smart home technologies is not borne out in practice."

(Wilson, Hargreaves and Hauxwell-Baldwin, 2017) Home automation and energy: what do we know?

With co-authors, I have written a number of times about different smart home technologies' propensity to lower energy consumption. Per-room, automated heating systems (Scott et al. 2011) can either raise or lower demand, depending on the occupancy of rooms in the house, and the settings of the previous control system (usually thermostats and/or thermostatic radiator valves). The industry is fond of claiming large savings compared to always-on heating and cooling - and a surprising amount of homes do not utilise any programmable function. However, we've previously pointed out (Hazas and Strengers 2019), that compared to a 
properly programmed thermostat, the makers of the Nest learning thermostat report a heating savings of about 7\%, and negligible savings for cooling (Nest Labs 2015, p. 11).

Smart lighting which responds to occupancy (real or anticipated), is another smart home technology with the potential to save energy. However, previous work has argued that smart lighting is most often deployed to create "a luxurious aesthetic experience" (Strengers and Nicholls, 2017, p. 2); this is part of a wider aspiration in the smart home industry to create technology-enabled "pleasance" (Strengers et al. 2020). Smart lighting often includes supplemental or feature lighting, and mood lighting which might be configurable to pre-set "scenes" or to change at different times of day. If control of the light is via an app (as is common with many Wi-Fi-enabled smart light bulbs), then there is additional, constant energy consumption due to networked standby; this can comprise half the electricity demand of the light bulb, with the other half used to actually generate light (Hazas and Strengers 2019).

Finally, occupancy or activity-based smart digital media and appliance control also has some potential for lowering demand - again, taking care that networked standby does not wipe out the savings. However, with these systems, normally the focus is on performance (high fidelity video and audio), interoperability, and convenience. And where devices must be controlled and tied together using off-the-shelf or bespoke home automation (e.g., smart plugs), then there is the additional embodied carbon (arising from manufacturing and shipping), as well as the standby energy drawn by those devices (Bates et al. 2013).

Just like thermal control, lighting, and digital media and appliance control, robotic vacuum cleaners and lawn mowers come attached with the touted bonus of saving energy; many analyses are posted online. However, can they really save energy? A more holistic discussion, which includes how these devices are meant to be used, and how they are used, is needed. 


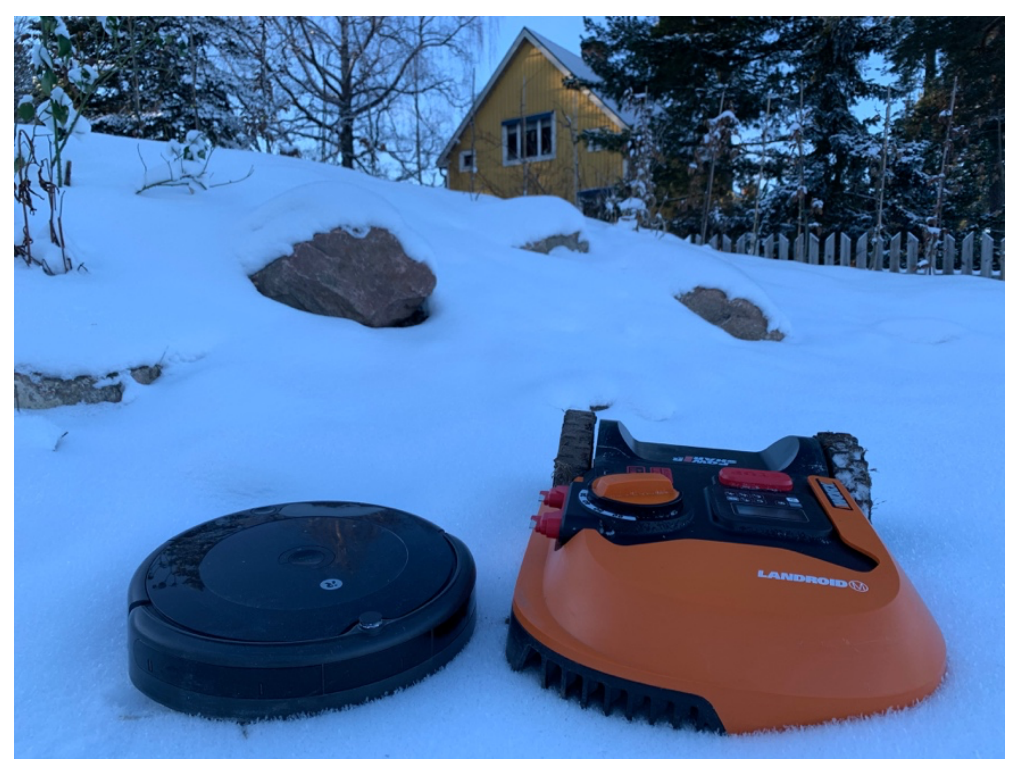

Figure 1: A front line in the battle against household energy demand? A robotic vacuum cleaner and lawn mower, out of their element.

\section{$\underline{\text { Robotic vacuum cleaners and lawn mowers }}$}

Robotic vacuum cleaners were introduced around 2002, and robotic lawn mowers were available from 1995, but not popularised until about 2010. Although one cuts and the other sucks, they work on similar principles. The simplest devices move forward until they "bump and turn": vacuums have bumper sensors and "cliff" detectors, and mowers instead rely on detection of an installed boundary cable. More complex (and expensive) models employ imaging and self-mapping. By running this way for a long period of time (at least several hours per week, but usually more), the robots can achieve good coverage of a floor or lawn. Both vacuums and mowers are becoming more commonplace, with market values measured in billions of US dollars, and their sales numbers are growing at an annual rate of up to $20 \%$.

Early on, there was interesting work examining how even these single-purpose, utilitarian robots effect new forms of interaction, and even change household dynamics. People quite quickly ascribed names, personalities, and mood swings to robotic vacuum cleaners (Forlizzi \& DiSalvo 2006; Sung et al. 2008). Fifteen years later, such findings would still seem to hold true. The iRobot Home app allows you to name connected Roombas, and one recent forum poster referred to their lawn mower as "Landroid Calrissian."

Impact comparison 
Many online estimates focus on the direct energy consumed by these devices, comparing them to their more traditional counterparts; often given alongside a cost comparison for purchase and perhaps maintenance. But if we are interested in looking at the total energy consumed, we must consider three key areas of energy consumption: the energy drawn for operation which includes charging and any standby or "parasitic" power; the embodied energy which includes manufacturing and transport as mentioned above, and also any spare parts for maintenance; and finally the energy for networking connectivity and data centre reliance.

Table 1 shows a reckoning for each of these categories. Embodied energy for production, transport and maintenance is notoriously difficult to estimate - there is an entire field of research devoted to this, called life cycle assessment. Rather than get the number wrong, I have reduced the assessment to "small", "medium" or "large." Generally speaking, things that contain metal and/or larger batteries tend to have a larger embodied impact. Alongside the embodied impact, I have given an indication of the potential lifespan: the robots have less embodied energy and carbon but tend not to last as long as their push-along cousins.

\begin{tabular}{|c|c|c|c|c|}
\hline & Embodied & $\begin{array}{l}\text { Potential } \\
\text { Lifespan }\end{array}$ & $\begin{array}{l}\text { Weekly direct } \\
\text { energy total } \\
\text { (of which } \\
\text { standby) }\end{array}$ & $\begin{array}{l}\text { Each app } \\
\text { update (size) }\end{array}$ \\
\hline $\begin{array}{l}\text { Conventional } \\
\text { vacuum, } 1400 \mathrm{~W}\end{array}$ & Medium & $10-20$ years & 1005 Wh (-) & - \\
\hline iRobot R692 & Small & $5-10$ years & $\begin{array}{l}504 \text { Wh }(210 \\
\text { Wh) }\end{array}$ & $\begin{array}{l}16 \text { Wh (336 } \\
M B)\end{array}$ \\
\hline Gas push mower & Large & $10-20$ years & Large (-) & - \\
\hline $\begin{array}{l}\text { Cordless electric } \\
\text { push mower }\end{array}$ & Large & $10-20$ years & $\begin{array}{l}520 \text { Wh }(200 \\
\text { Wh) }\end{array}$ & - \\
\hline $\begin{array}{l}\text { WORX Landroid M } \\
\text { WR142E }\end{array}$ & Medium & $5-10$ years & $\begin{array}{l}874 \text { Wh }(202 \\
\text { Wh) }\end{array}$ & $3 \mathrm{Wh}(70 \mathrm{MB})$ \\
\hline
\end{tabular}

Table 1: Impact comparison for vacuum cleaners and lawn mowers. Assumptions: Conventional vacuum and lawn mower run for 45 minutes, once per week. Robots run every day for one charge (approximately 90 mins of operation for the above models). Internet data traffic used for robot settings and operation is minimal. App downloads have an energy footprint of 50 Wh per gigabyte (includes Wi-Fi, broadband, Internet, data center). Robot standby figures based on direct measurement. 
In direct energy consumption, we see some quite similar figures. A robotic vacuum cleaner might consume about half of what a conventional one does. Even if the robots do not run every day, a significant component is the standby energy for the charging base, which is about 200 watt-hours per week. And note that earlier-model Roombas registered a much higher standby (three watts); in this case, the majority of consumption was in standby. (For a wonderfully detailed analysis of robotic vacuum cleaner power consumption, including standby, charging, and different robot subsystems in operation, see Vaussard et al. (2014).) In all, despite being much smaller, more efficient devices, the robots have comparable energy consumption because the design assumption is that they can (and should) run much more often. However, I should point out that substituting a robotic lawn mower for a gas-powered push mower is a clear win; the electric motor is simply much more efficient than an internal combustion engine.

Out of the box, these devices work in a basic way, normally via a console pushbutton, without connecting to the cloud. However, they tend to be easier to control and configure, if connected and accessed using a companion app. The typical design assumption is that a device gets connected; quick start guides include the app name and basic setup instructions, and sometimes a QR code that links to the app store. Registration in the app not only links the app to the robot, but also enables the robot itself to communicate with the local Wi-Fi. Giving these "smart" systems the benefit of the doubt, I assume here that the connectivity and data centre activity required to reconfigure or control the robot is minimal, although it will have some finite footprint that could be estimated by profiling its traffic. (And profiling would answer the question, "Does the service robot connect only when I access it from the app, or does it phone home to the data center more often?") Larger software updates (as is the case with iRobot Home), particularly if applied often, could be a source of energy demand, but likely much less than charging and standby.

\section{Raising the level of service provision}

Comparison tables like the one above imply that service robots are a simple, one-to-one swap for more conventional appliances. And yet, the prevailing evidence is that these robots tend supplement rather than wholly replace (Sung et al. 2008; Nicholls and Strengers 2019). This is particularly the case with vacuums: a conventional vacuum (usually the old appliance) is periodically used to spot clean, or finish off the work a robotic vacuum didn't do in a particular session. And, work by robotic mowers needs to be supplemented by an electric string trimmer for edges and hard to reach places, or (better for energy demand) manual garden shears. 
Energy savings aside, a perceived benefit is that these robots raise the level of provision: a nicelooking lawn; a consistently particle-free floor. As mentioned earlier, they have an inscribed way of being used, through default settings and manufacturer recommendations. There is a strong assumption that they should run at least several times per week: apps allow the multiple sessions to be enshrined in a weekly schedule. For example, the WORX Landroid models, out of the box, run five days per week, Monday through Friday. The goal of frequent operation is to achieve even coverage of the area to be mowed or cleaned. But another reason is that frequent sessions render achievable the lofty expectations for cleanliness and tidy presentation that many householders have come to pursue (Nicholls and Strengers 2019). If you rely on a domestic service robot like these, one message is that we should be aware of, but not to get too hung up on, the savings or waste they incur. Unless you are retiring a gaspowered mower, these are small potatoes. For households, the watt-hour quantities in Table 1 are dwarfed by the energy and carbon emissions associated with the provision of food, heating, cooling, hot water, and transportation. If you're concerned about energy demand and the environment, then work on eating less meat, adjust your thermostat (in the winter, put on a sweater; in the summer, have iced drinks to keep cool), and ride a bike, bus or drive an electric vehicle rather than a gas or hybrid car.

As with smart lighting, thermostats, and digital media and appliance control, these service robots do not necessarily result in significant energy savings. And, they come with the caveat that in typical use, they remain supported by traditional appliances. As we continue to make "smart" or automate other areas of domestic life, it is important to keep an eye on the impacts. Remember Amazon Dash? Millions of these stand-alone buttons were manufactured and shipped to households, to enable an instant (and some argue, mindless) re-order of consumables, from wet wipes to batteries. Amazon stopped supporting Dash buttons in 2019. All too often with innovation for smart homes, the dialogue is about convenience, pleasance and fidelity; while environmental impacts are often a touted benefit, they are difficult to empirically justify.

Mike Hazas is Professor of Human-Computer Interaction at Uppsala University. Contact him at mike.hazas@it.uu.se.

\section{REFERENCES}


J. Forlizzi and C. DiSalvo, "Service robots in the domestic environment: a study of the roomba vacuum in the home," in Proceedings of the 1st ACM SIGCHI/SIGART conference on Humanrobot interaction, 258-265, 2006. DOI:https://doi.org/10.1145/1121241.1121286

O. Bates and M. Hazas, "Exploring the hidden impacts of HomeSys: Energy and emissions of home sensing and automation," In the Adjunct Proceedings of the 2013 ACM Conference on Pervasive and Ubiquitous Computing, pp. 809-814, 2013.

DOI:https://doi.org/10.1145/2494091.2497322

M. Hazas and Y. Strengers, "Promoting smart homes," in Energy Fables: Challenging Ideas in the Energy Sector, J. Rinkinen, E. Shove, J. Torriti (eds.), pp. 78-87, 2019.

Nest Labs, Energy savings from the Nest Learning Thermostat: Energy bill analysis results, [online], 2015. Available at: http://downloads.nest.com/press/documents/energy-savingswhite-paper.pdf. Accessed 4 Feb 2021.

L. Nicholls, Y. Strengers, "Robotic vacuum cleaners save energy? Raising cleanliness conventions and energy demand in Australian households with smart home technologies," Energy Research \& Social Science 50, pp. 73-81, 2019. https://doi.org/10.1016/j.erss.2018.11.019.

J. Scott, A.J. Brush, J. Krumm, B. Meyers, M. Hazas, S. Hodges, N. Villar, "PreHeat: controlling home heating using occupancy prediction," In Proceedings of the 13th International Conference on Ubiquitous Computing, pp. 281-290, 2011. https://doi.org/10.1145/2030112.2030151

Y. Strengers, M. Hazas, L. Nicholls, J. Kjeldskov, M. Skov, “Pursuing pleasance: Interrogating energy-intensive visions for the smart home," International Journal of Human-Computer Studies, Academic Press Inc.. 136. 2020.

Y. Strengers, L. Nicholls, "Convenience and energy consumption in the smart home of the future: Industry visions from Australia and beyond," Energy Research \& Social Science, 32, pp. 86-93, 2017.

Ja-Young Sung, Rebecca E. Grinter, Henrik I. Christensen, and Lan Guo. 2008. Housewives or technophiles? understanding domestic robot owners. In Proceedings of the $3 \mathrm{rd}$ ACM/IEEE international conference on Human robot interaction (HRI '08). Association for Computing Machinery, New York, NY, USA, 129-136. DOI:https://doi.org/10.1145/1349822.1349840

F. Vaussard, J. Fink, V. Bauwens, P. Rétornaz, D. Hamel, P. Dillenbourg, F. Mondada, "Lessons learned from robotic vacuum cleaners entering the home ecosystem," Robotics and Autonomous Systems, Volume 62, Issue 3, pp. 376-391, 2014.

https://doi.org/10.1016/j.robot.2013.09.014. 
C. Wilson, T. Hargreaves, R. Hauxwell-Baldwin, "Benefits and risks of smart home technologies", Energy Policy, Volume 103, pp. 72-93, 2017. https://doi.org/10.1016/j.enpol.2016.12.047. 